\title{
Non-Rayleigh distribution of reflected speckle intensities from localized states inside the gap of disordered photonic crystals
}

\author{
Xiangdong Zhang and Zhao-Qing Zhang* \\ Department of Physics and Institue of Nano Science and Technology (INST), The Hong Kong University of Science and Technology, \\ Clear Water Bay, Kowloon, Hong Kong, China
}

(Received 13 November 2001; revised manuscript received 22 April 2002; published 21 June 2002)

\begin{abstract}
The field distributions of reflected speckles arising from localized states inside the gap of disordered photonic crystals in two dimensions were studied through numerical simulations using the multiple-scattering method. The statistics of the Lyapunov exponent of the transmitted waves were also studied. Similar to the case of disordered photonic crystals in one dimension, two types of localized states were found depending on the degree of disorder and the frequency inside the gap. Our simulation results indicate that the reflection statistics depend on whether or not the localized states are of the normal type. They also depend on whether the reflected angles are in the Bragg direction or not. For the non-Bragg angles, we found that the intensity distribution arising from the normal-type localized states follows Rayleigh statistics, in agreement with random matrix theory. However, deviations from Rayleigh statistics were found for second type of localized states. The crossover behavior from non-Rayleigh to Rayleigh statistics was studied as a function of the degree of disorder. By separating the field into coherent and diffuse parts, we have studied the statistics of field and phase distributions for both diffuse and total fields as well as their speckle contrasts. It is found that the crossover behavior is very similar to behavior in ballistic to diffusive wave propagation for the transmitted waves and can be described by the random-phasor-sum model (RPS). For the Bragg angle, non-Rayleigh statistics were found for both kinds of localized states. The statisics are sensitive to the degree of disorder. It is found that both the RPS and $K$ distribution have limited ranges of validity in this case.
\end{abstract}

DOI: 10.1103/PhysRevB.65.245115

PACS number(s): 42.25.Bs, 72.15.Rn, 42.25.Dd

\section{INTRODUCTION}

In the past few years, the scattering of both classical and quantum waves from random media has been the focus of intensive study. ${ }^{1}$ A wave undergoes multiple scatterings due to the presence of imhomogenieties or impurities as it propagates through a random medium. As a result, the scattered intensities become highly irregular. Those speckle pattern should be described in statistical terms. There has been considerable study of the statistics of transmitted speckle intensities in random media. ${ }^{1-9}$ It is well known that, in the absence of interference, transmitted speckle intensities follow Rayleigh statistics (a negative exponential law). The presence of interference always produces an anomalous exponential tail. ${ }^{3-5}$ In a strong scattering medium, the distribution can cross over to lognormal behavior when waves become localized. ${ }^{8,9}$ Thus, a strong deviation from Rayleigh statistics is an indication of localization.

Recently, interest has shifted to studying speckle intensity distributions in reflection geometry. Contrary to the intensity of transmitted speckles, random matrix theory has predicted that, in a waveguide with large number of channels, the intensity of reflected speckles follows Rayleigh statistics even in the localized regime. ${ }^{10}$ In other words, the characteristics of localized states present in a random medium are not captured in reflection statistics. This result is rather interesting.

The physical origin of these two distinct transmission and reflection behaviors may be understood in the following way. Consider a localized sample with a thickness larger than the localization length. In the transmission case, since most of the incident waves are reflected due to wave localization, the transmitted waves that appear at the surface have, in large part, been mediated by some localized state located near the center of the sample. These waves experience many recurrent scatterings around the localized state and have long path lengths. Because of the large spatial intensity fluctuations of the localized state, the transmitted speckles show large intensity fluctuations, leading to a distribution that is very different from Rayleigh statistics. However, the situation is different in the reflection case. Since the source is close to the input surface (a distance on the order of the scattering mean free path), the dominant contributions to the reflected speckle intensities actually come from waves that have shorter path lengths. These multiply scattered waves do not self-intersect and, therefore, show no interference effects. Any waves with longer path lengths that can probe a localized state through recurrent scatterings now carry a much smaller weight in the reflected intensity. Thus, in the reflection geometry, the characteristics of localized states are completely overwhelmed by the presence of nonintersecting paths and, therefore, cannot be captured in the reflection statistics.

The localized states discussed above are the normal-type localized states, i.e., the Anderson localized states, which are caused by the interference effects in strongly scattering media. ${ }^{1}$ However, it has been shown that there is another type of localized states lying inside the band gaps of disordered photonic crystals. ${ }^{11,12}$ Photonic crystals themselves have attracted a lot of attention in recent years. ${ }^{11-16}$ For frequencies inside a band gap, waves are evanescent and cannot propagate in those crystals. When disorder is introduced, localized states can appear inside a gap and, therefore, reduce the size of the gap. ${ }^{14-16}$ With the increase of the degree of disorder, more localized states appear in the gap. When the 
density of states is sufficiently large, the gap may be destroyed completely and the wave propagation may lose its evanescent nature for the entire gap. Thus, a disordered photonic crystal is a good system for studying strong localization states. Recent investigation of transport behavior in disordered photonic crystal in one dimension shows that there can exist two types of localized states inside a band gap. ${ }^{11,12}$ In the case of large disorder, all the states inside the gap belong to the normal-type localized states, satisfying the singleparameter scaling theory. ${ }^{13}$ However, when the disorder is small, the localized states well inside a gap do not satisfy the single-parameter scaling theory due to the residual evanescent nature of the waves. The spatial extent of such localized states are, in general, smaller than that of the normal type. Although the existence of two types of localized states is only established in one dimension, it is expected that this is also true in higher dimensions. In fact, the strongly localized states have been observed inside the gap of disordered photonic crystals in both two and three dimensions. ${ }^{15,16}$

A natural question to ask is what would be the statistics of the reflected speckles arising from the second type of localized state? In order to answer this interesting question, in this work, by using the multiple-scattering method, we have carried out extensive numerical simulations of the reflected speckles which are produced by the localized states inside the gap of two-dimensional disordered photonic crystals. In order to establish the existence of two types of localized states in two dimensions, we have also studied the statistics of the Lyapunov exponent of the transmitted waves. Similar to the case of one dimension, two types of localized states were found depending on the degree of disorder and the frequency position inside the gap. Indeed, our simulation results of reflected speckles indicate that the reflection statistics depend on whether or not the localized states are of the normal type. They also depend on whether or not the reflected angles are in the Bragg direction. In non-Bragg angles, we found that the intensity distribution arising from the normal-type localized states follows Rayleigh statistics, in agreement with random matrix theory. ${ }^{10}$ However, deviations from Rayleigh statistics were found in the case of the second type of localized states.

In order to understand the crossover behavior from a nonRayleigh to Rayleigh statistics, we have studied the change in statistics as a function of the degree of disorder at a fixed frequency inside the band gap. By separating the field into the average (coherent) and residual (diffuse) parts, we have studied the statistics of field and phase distributions for both diffuse and total fields. It is found that their statistics can be described by a random-phasor-sum (RPS) model. ${ }^{17-19} \mathrm{We}$ have also studied the speckle contrast (SC) for both parts. ${ }^{17,19}$ All these results indicate that the crossover behavior from a non-Rayleigh to Rayleigh statistics is very similar to that of ballistic to diffusive wave propagation in transmitted waves. However, for the Bragg angle, only non-Rayleigh statistics were found for both types of localized states. This is a result of single mode exciation with a large coherent part in the Bragg scattering angle. The reflection statisics are sensitive to the degree of disorder. At large randomness, the statistics of both the average and residual fields can be de- scribed by the RPS. When the degree of randomness is reduced, it is found that the RPS cannot describe the distribution of both fields. However, the residual field can be described by $K$ distribution as a result of incomplete phase randomization. ${ }^{19}$ As the randomness is further reduced, we find that even $K$ distribution fails to describe the distribution of the residual field.

The rest of this paper is arranged as follows. In Sec. II, we define the system and introduce the method of numerical simulation. The results and discussion are described in Sec. III. A conclusion is given in Sec. IV.

\section{SYSTEM AND METHOD OF SIMULATION}

The two-dimensional (2D) photonic crystals considered here consist of a square array of dielectric cylinders in an air background. The cylinders have the same radius $R$ and dielectric constant $\epsilon=11.4$. The Maxwell equation for the $s$-polarized waves takes the form $\left[c^{-2} \omega^{2} \epsilon(\vec{r})+\nabla^{2}\right] E(\vec{r})$ $=0$, where $E(\vec{r})$ is the electric field along the cylinder axis and $\epsilon(\vec{r})$ is the position-dependent dielectric constant. It has been shown that complete gaps exist for a certain range of $R / a$, where $a$ is the lattice constant. ${ }^{13}$

Disordered photonic crystals were produced by randomizing an ordered one. The degree of randomness was controlled. Consider two cases: small randomness and complete randomness. For the case of complete randomness, the sample was produced by randomly altering the position of each cylinder within a distance $(a-2 R) / 2$. The procedure was repeated 1000 times to ensure complete randomization. A move was forbidden if two cylinders overlapped. For the case of small randomness, each cylinder was moved randomly, but only once within a range $[-d r, d r]$, where $d r$ $<(a-2 R) / 2$.

The transmission, reflection, and scattering properties of the disordered photonic crystals were calculated using the multiple-scattering method. ${ }^{20}$ The multiple scattering method is best suited for a finite collection of cylinders with a continuous incident wave of fixed frequency. For circular cylinders, the scattering property of the individual cylinder can be obtained analytically, relating the scattered fields to the incident fields. The total field, which includes the incident plus the multiple-scattered field, can then be obtained by solving a linear system of equations, whose size is proportional to the number of cylinders in the system. Both near field and far field radiation patterns can be obtained straightforwardly. In two dimensions, we note that there exist straight rows of cylinders in both the $x$ and $y$ directions. The reflected intensities in the far field can be expressed as $I(\theta)=|f(\theta)|^{2} / \rho$, where

$$
\begin{aligned}
f(\theta)= & \sqrt{\frac{2}{\pi k_{0}}} e^{-i \pi / 4} \sum_{i=1}^{N} \sum_{m=-M}^{M} e^{-i k_{0} \rho_{i} \cos \left(\theta_{i}-\theta\right)} \\
& (-i)^{m} e^{i m \theta} B_{m}(i) .
\end{aligned}
$$

Here $B_{m}(i)$ represent the expansion coefficients of the radiated waves at the $i$ th cylinder, which is determined by the self-consistent equation described in Ref. 20. $k_{0}=\omega / c$, and 


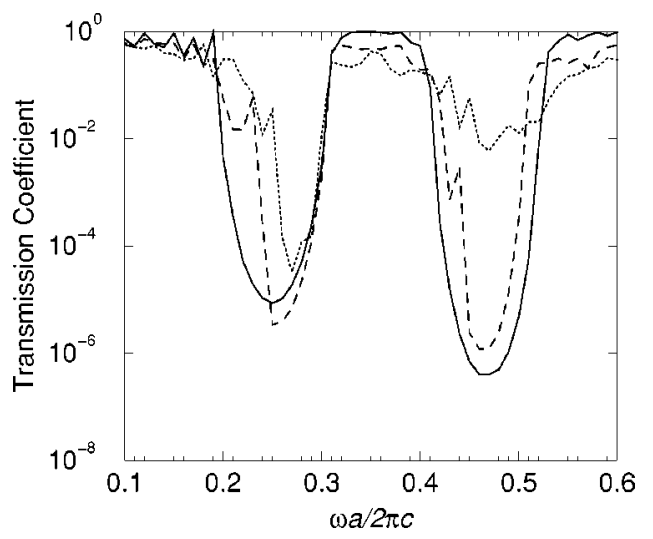

FIG. 1. Transmission coefficients for ordered and disordered photonic crystals represented by a square lattice with dielectric cylinders of radius $R=0.3 a$ and $\epsilon=11.4$. The solid line is the ordered case; the dashed line is the case of small randomness with $d r$ $=0.2$; dotted line is the case of complete randomness.

$\vec{\rho}_{i}=\left(\rho_{i}, \theta_{i}\right)$ denote the site coordinates of the $i$ th scatterer. $M=\infty, N$ is the amount of scatterer. $\vec{\rho} \equiv(\rho, \theta)$ specifies the detected position in a two-dimensional plane. Thus, the statistics of reflected intensities at various angles can be determined by the statistics of $|f(\theta)|^{2}$ when properly normalized. The source is prepared by passing a plane wave through an open slit in front of the sample. The width of the slit is about $20 \%$ smaller than the sample width to avoid the scattering at the sample edges. The position of the slit is about two lattice constants in front of the sample. In this case the incident field can be obtained from the Kirchoff integral formula. For a plane wave, $\exp \left(i k_{0} x\right)$, incident from $x<0$, the diffracted wave in the region $x>0$ arising from a slit centered at the origin with an opening of width $w$ in the $y$ direction is given by

$$
u_{\mathrm{inc}}(x, y)=\left(\frac{k_{0}}{4}\right) \int_{-w / 2}^{w / 2} d y^{\prime}\left[H_{0}\left(k_{0} \rho^{\prime}\right)+i \frac{x}{\rho^{\prime}} H_{1}\left(k_{0} \rho^{\prime}\right)\right],
$$

where $\rho^{\prime}=\sqrt{x^{2}+\left(y-y^{\prime}\right)^{2}}$, and $H_{m}$ is the Hankel function of the first kind. Such a method is a very efficient way of handling the scattering problem of a finite sample containing cylinders of circular cross sections in an arbitrary arrangement, and this method is easy to realize in experiments. The detailed description of this method has been given in Ref. 20.

\section{NUMERICAL RESULTS AND DISCUSSION}

In our calculations, a sample was chosen of width $W$ and thickness $L$, denoted as $W \times L$. The source was prepared by passing a plane wave through an open slit in front of the sample. In order to ensure sufficient angular resolution for the study of reflected statistics, $W$ should be sufficiently large. In all the calculations discussed below, $W$ was chosen to be 101a. In an ordered system, the transmission coefficient $T$ as a function of the renormalized frequency $f$ $(=\omega a / 2 \pi c)$, for a sample of size $101 \times 7$ along the $\Gamma-X$ direction is plotted in Fig. 1 as a solid line for $R=0.3 a$.
There exist two gaps for frequencies below $f=0.6$. Inside these two gaps, the wave propagation is evanescent in nature.

When randomness is introduced, localized states may appear inside the gap and the gap size reduces accordingly. In the case of small randomness with $d r=0.2$, the transmission spectrum of one particular random configuration is shown as a dashed line in Fig. 1. Inside each gap, there is a peak near the lower band edge. This indicates the existence of some localized states at the peak frequencies. The presence of localized states reduces the size of a gap and, therefore, suppresses the evanescent nature of the waves inside the gap.

In the case of complete randomness, the transmission spectrum of one particular random configuration is shown as a dotted line in Fig. 1. In this case, more localized states appear deeper inside the gap. As a result, the first gap is further reduced, whereas the second gap is now completely destroyed and the wave propagation loses its evanescent nature.

A quantitative way to correlate the effects of randomness on the evanescence of waves is to study the statistics of the Lyapunov exponent. ${ }^{11}$ A Lyapunov exponent $\lambda$ is defined as the inverse of the decay length of the transmission amplitude. In the region where the density of states is zero, it is equivalent to the inverse of the decay length of the evanescent waves. In a region where the density of states is nonzero, it is equivalent to the inverse of the localization length of the localized states. In general, the Lyapunov exponent can be defined as $T \propto \exp (-2 L \lambda)$ at the limit of infinite $L$. For a finite-sized sample, the Lyapunov exponent may fluctuate from sample to sample due to the random occurrence of localized states inside the gap. Thus, it is convenient to study the variance of the Lyapunov exponent, i.e., $\operatorname{var}(\lambda)=\left\langle\lambda^{2}\right\rangle$ $-\langle\lambda\rangle^{2}$. $^{11}$

For the frequencies inside the two gaps shown in Fig. 1, we have simulated the Lyapunov exponents from a sample of size $101 \times 12$ with 5000 different configurations for each frequency. The average $\lambda$ and its variance as a function of frequency are plotted in Fig. 2 as circles and squares, respectively. Figures 2(a) and 2(b) represent the results with small randomness with $d r=0.2$ and complete randomness, respectively.

In the case of complete randomness [Fig. 2(b)], localized states appear everywhere inside the two gaps, as is evident from the fact that $\operatorname{var}(\lambda) \neq 0$ inside the gaps. Inside the high frequency gap, the density of states is sufficiently large that the dip in $\operatorname{var}(\lambda)$ disappears completely. Thus, the waves lose their evanesent nature completely. All the states inside this gap belong to normal-type localized states. In the low frequency gap, a residual dip persists due to the insufficient density of states. The presence of a dip in this case signifies the presence of residual evanescent waves. In the case of small randomness [Fig. 2(a)], localized states appear only near the band edges of the two gaps resulting in a nonzero $\operatorname{var}(\lambda)$. For frequencies well inside the gap, the density of states remains zero and the wave propagation is purely evanescent, independent of the configuration. This is indicated by a vanishing $\operatorname{var}(\lambda)$. Thus, as a function of frequency, $\operatorname{var}(\lambda)$ exhibits a dip inside each gap with two maxima located near the band edges. The presence of such a dip actu- 


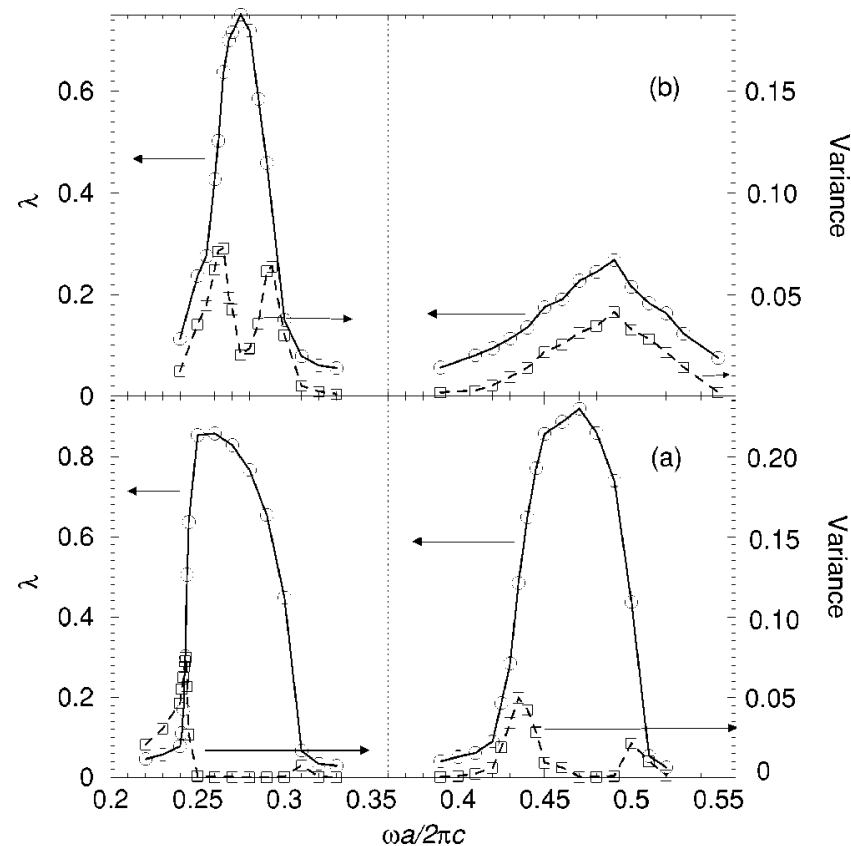

FIG. 2. Lyapunov exponent (circles) and its variance (squares) in the frequency ranges of the band gaps in Fig. 1 for the case of small randomness (a) and complete randomness (b).

ally signifies the presence of residual evanescent waves in the system. The localized states lying outside the dip belong to the normal type, whereas those lying inside the dip belong to the second type. Since the second type of localized states appear well inside the gaps, they have much smaller localization lengths. Our results shown in Fig. 2 are very similar to those found in one-dimensional cases. ${ }^{11}$ It should be pointed out that even in the case of complete randomness, the density of the localized states inside the gaps is not constant. This is indicated by the presence of a peak in $\lambda$ near the center of each gap shown in Fig. 2(b). A peak in $\lambda$ implies a dip in the decay length, which in turn implies a dip in the density of states. Thus, complete randomization does not wash out all the underlying features of the photonic crystal. This is due to the presence of hard core repulsions among the scatterers. In other words, systems possess certain shortrange order even when they have been completely randomized. As a result of such short-range order as well as the the presence of Mie resonances, some variations in the density of states still remain.

Before we present the simulation results of the speckle statistics in the reflection geometry, we show in Fig. 3 a typical reflected intensity distribution as a function of scattered angles in the absence of disorder for a normally incident wave at some frequency inside the gap. The large intensity at $\theta=180^{\circ}$ is a result of Bragg scattering. The existence of small oscillations at the non-Bragg angles is due to wave diffraction as a result of the finite beam width of the incident wave. For frequencies inside a pass band, the reflected intensity will be much reduced as most of the waves can propagate through the sample. When randomness is introduced, the scattered waves can appear at any angle. Thus, it is in-

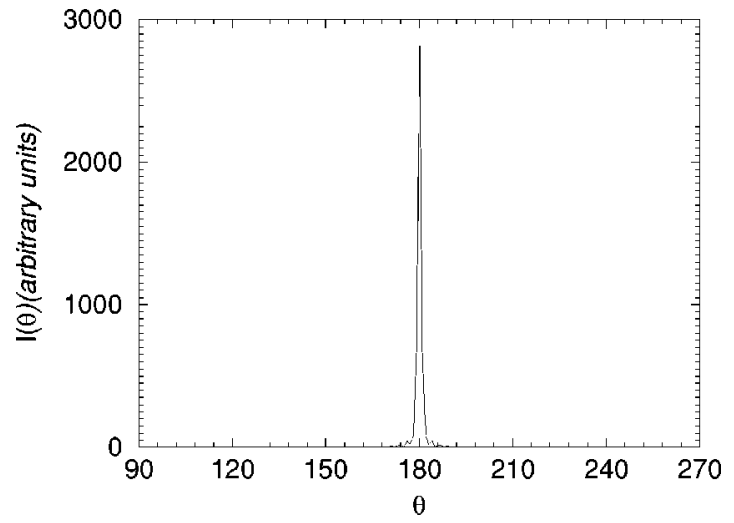

FIG. 3. The reflected intensity distribution as a function of scattered angles for ordered photonic crystals of a square lattice with $R=0.3 a$ and $\epsilon=11.4$ at $f=0.44$. The solid line is the ordered case; the dashed line is the case of small randomness with $d r=0.2$; the dotted line is the case of complete randomness.

teresting to study the speckle statistics for both the Bragg and the non-Bragg angles.

We first discuss the case of the non-Bragg angles. For each configuration, we calculate the intensity at ten different angles between $160^{\circ}$ and $170^{\circ}$. The process was repeated for 5000 configurations. The results of our simulations indicate that the normalized speckle intensity distribution $P(I /\langle I\rangle)$ that arises from the normal-type localized states follows Rayleigh statistics. To show this, in Fig. 4(a), we plot the normalized speckle intensity distribution $P(I /\langle I\rangle)$, for the following four frequencies: (i) $f=0.220$ (upper triangles), (ii) $f=0.430$ (squares) for the case of small randomness, (iii) $f=0.250$ (lower triangles), and (iv) $f=0.480$ (circles) for the case of complete randomness. The straight solid line denotes

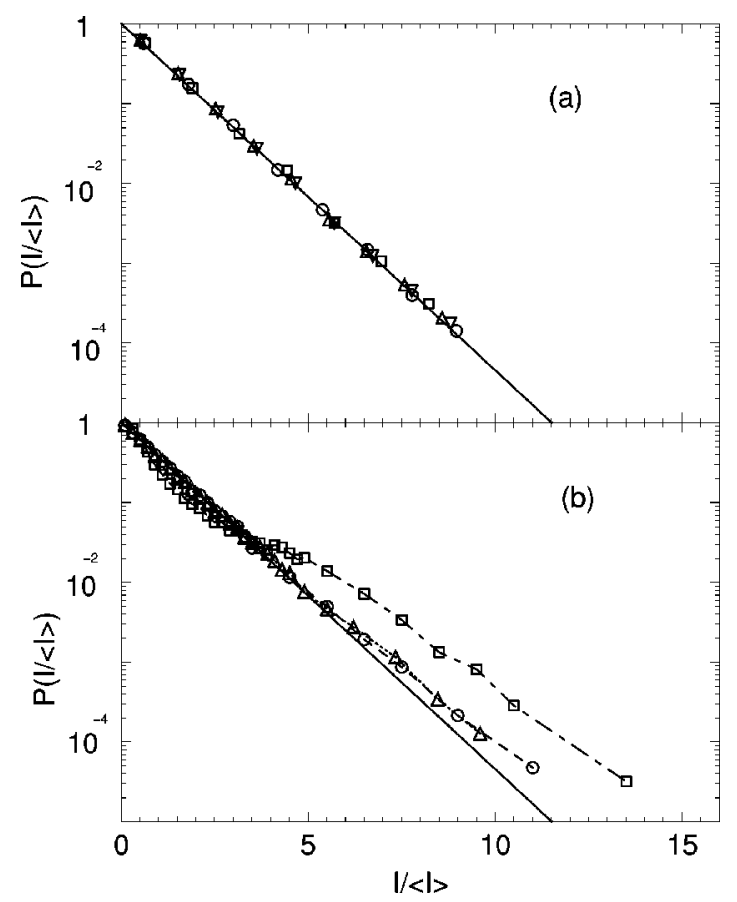

FIG. 4. $P(I /\langle I\rangle)$ as a function of $I /\langle I\rangle$ arising from (a) normal type localized states and (b) the second type localized states. 
a simple exponential decay. It is clear that all distributions follow Rayleigh statistics. Frequencies in (i)-(iii) are located outside the three dips shown in Fig. 2, but close to the lower band edges. Whereas, the frequency in (iv) lies near the middle of the second gap. In this case, the gap is completely filled by localized states and the evanescent nature of the gap is lost entirely. These results are in good agreement with the prediction of random matrix theory. ${ }^{10}$ However, we find that the distribution of $P(I /\langle I\rangle)$ that arises from the second type of localized states exhibits a non-Rayleigh statistics. To show this, we plot in Fig. 4(b) the function $P(I /\langle I\rangle)$ for the following three frequencies: (i) $f=0.244$ for the case of small randomness (squares), (ii) $f=0.445$ for the case of small randomness (circles), and (iii) $f=0.275$ for the case of complete randomness (triangles). Each frequency lies inside the dip of $\operatorname{var}(\lambda)$ shown in Fig. 2, but close to the lower band edges. Clearly, they all exhibit non-Rayleigh statistics. The deviation from the Rayleigh distribution is the largest in the first gap when the randomness is small. This is also the case where the evanescent waves predominate.

Here, the interesting question to ask is how the transition from the non-Rayleigh to the Rayleigh distribution occurs in the statistics of the reflected speckle intensities. In order to study the crossover behavior, we have chosen a fixed frequency, say $f=0.44$, in the second gap, and we have studied the field distribution as a function of the degree of disorder. In the case of completely disorder, the intensity distribution is of Rayleigh type as indicated in Fig. 2(b). However, at small randomness with $d r=0.2$, the distribution becomes non-Rayleigh as indicated in Fig. 2(a).

In fact, the transition from the non-Rayleigh to the Rayleigh distributions has been observed in microwave experiments on the crossover region of the ballistic to diffusive wave propagation of transmitted waves. ${ }^{18}$ In this study, the authors separated the field into the average (coherent) and the residual (diffuse) parts and studied the amplitude and phase distributions of both the residual and total fields. When sample thickness $L$ is smaller than the scattering mean free path, the presence of the large coherent part in the transmitted waves makes the total intensity distribution nonRayleigh. When $L$ increases to a few scattering mean free paths, it is expected that the Rayleigh distribution will be restored due to the negligible contribution of the coherent intensity. The RPS that considers the wave field as a sum of a circular random phasor and a constant coherent part with results derived from two kinds of diagrams has been successfully used to describe the change in statistics in such transitions. ${ }^{18,21}$ However, in the ballistic limit when the coherent part dominates, the amplitude distribution of the total field was found to deviate slightly from the the Gaussian distribution predicted by the RPS. Nevertheless, it was found that the distribution of the residual field is close to the Gaussian distribution.

Another interesting statistical quantity to be mentioned is the speckle contrast (SC), which is defined as $\mathrm{SC}(I)$ $=\sigma_{I} /\langle I\rangle\left[\right.$ with $\left.\sigma_{I}^{2} \equiv \operatorname{var}(I)\right]{ }^{17,19}$ When SC is one, the intensity distribution is of the Rayleigh type. Deviation from one implies a non-Rayleigh distribution. It has been pointed out that in the case of the residual field, a deviation from $\mathrm{SC}=1$

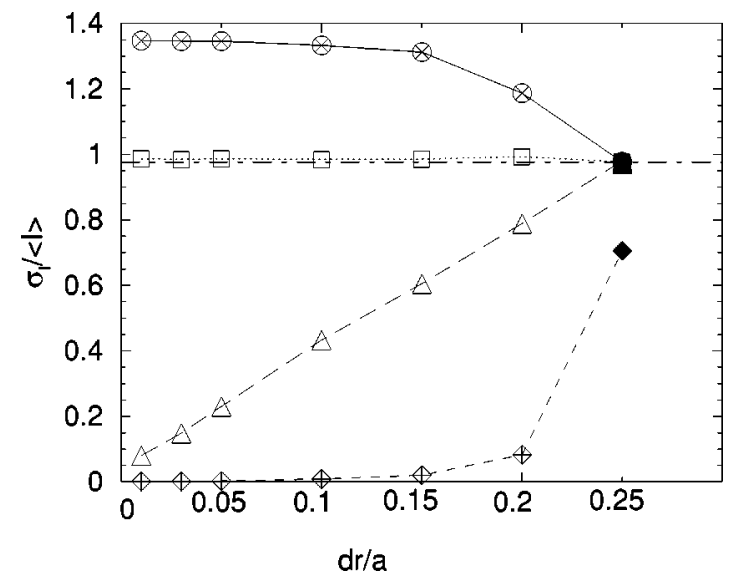

FIG. 5. Speckle contrast (SC), $\sigma_{I} /\langle I\rangle$, as a function of disorder degree. The solid line (circle) and The dashed line (diamond) are for the residual field and the total field in the Bragg direction; Dotted line (square) and long-dashed line (triangular) for the residual field and the total field in non-Bragg direction. The corresponding filled points are for the complete random case.

indicates that the phase randomization is not complete and speckles are not completely developed. In this case, the distribution function of the residual field should be governed by $K$ distributions. ${ }^{19}$ This situation is more likely to occur when the system is excited by a single mode. In this case, the phase fluctuations in the residual field are mainly due to those from the incoming mode, therefore making phase randomization difficult. ${ }^{18,19}$

In our work, we find it convenient to adopt the theoretical tools used in Refs. 18 and 19 to analyze our simulation data. Thus, we write the complex field $E(\theta)$ as the sum of the average and the residual fields

$$
E(\theta)=\langle E(\theta)\rangle+\delta E(\theta),
$$

where the angle brackets represent the ensemble average over different disorder realizations. The coherent and residual intensities can be written as $I_{c}=|\langle E(\theta)\rangle|^{2}$ and $I_{\text {res }}$ $=|\delta E(\theta)|^{2}$, being the averaged total intensity $\langle I\rangle=\left\langle I_{\text {res }}\right\rangle$ $+I_{c}$. In this work, we will discuss the statistical properties of both $E(\theta)$ and $\delta E(\theta)$.

In order to study the transition from the non-Rayleigh to the Rayleigh distributions in the reflected speckle intensities, we first study the behavior of SC as a function of the degree of disorder. For the non-Bragg angles, we calculate the scattered intensities at 20 different angles between $155^{\circ}$ and $175^{\circ}$ for each configuration. The process is repeated for 8000 configurations. The sample width in all the calculations presented below is taken as 8a. For both the residual intensity $\left[\mathrm{SC}\left(I_{\text {res }}\right)\right]$ and the total intensity $[\mathrm{SC}(I)]$, our simulation results show very similar behavior as a function of the degree of disorder for different angles. However, as a function angle, there exist some oscillations in the values of $\operatorname{SC}\left(I_{\text {res }}\right)$ and $\mathrm{SC}(I)$ due to the presence of oscillations in the coherent part. Here, we only show the behavior of $\operatorname{SC}\left(I_{\text {res }}\right)$ and $\mathrm{SC}(I)$ for the case of $\theta=174^{\circ}$ in Fig. 5 by open squares and open triangles, respectively. It should be pointed out that the filled square and filled triangle represent the case of complete ran- 

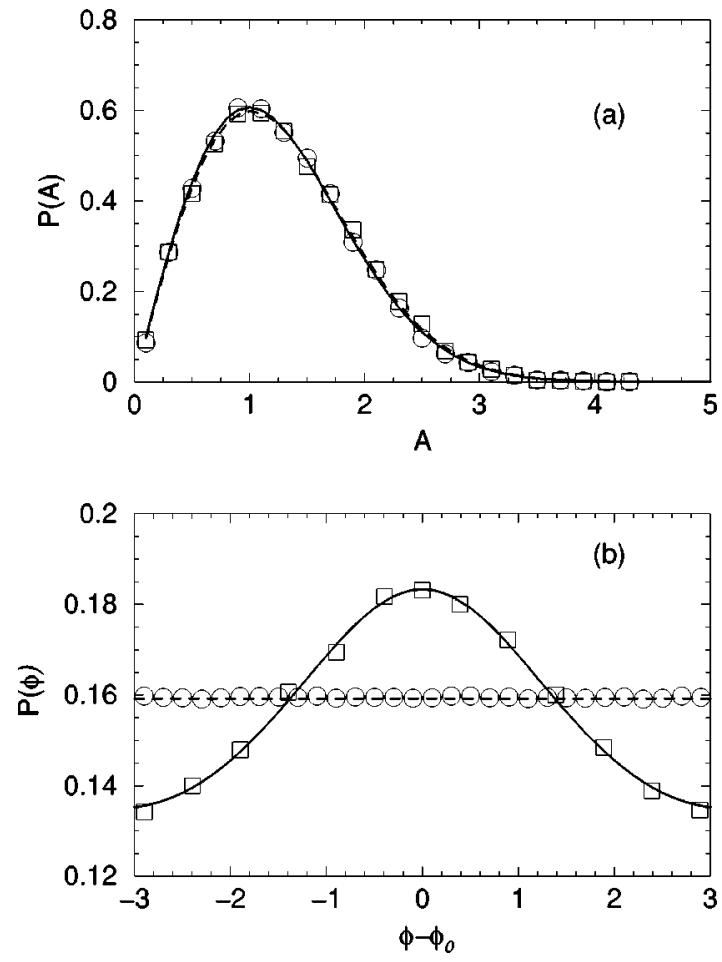

FIG. 6. Distributions of the normalized amplitude (a) and phase (b) of the total field (squares) and the residual field (circle) in the non-Bragg direction for the completely random case with $R=0.3$ and $f=0.44$. Solid lines and dotted lines are the theoretical predictions using the random-phasor-sum model.

domness. They do not represent the case of $d r=0.25$. It is interesting to point out that the monotonic increase of $\mathrm{SC}(I)$ as a function of the degree of disorder is similar to what has been found in the SC of the speckle pattern as a function of surface roughness. ${ }^{17}$ Also in Fig. 5, we show the corresponding results at the Bragg angle, i.e., $\theta=180^{\circ}$, by circles and diamonds. The large differences in SC between the Bragg and non-Bragg angles are obvious. We have also performed the simulation with an oblique incidence with an incident angle $45^{\circ}$ to the surface normal of the sample. Similar results have been found. We also plot these results as crosses and pluses in Fig. 5. They completely overlap with the results of normal incidence. Thus, the statistical properties of the reflected speckle intensities do not depend on the angle of incident. Since the behaviors of $\operatorname{SC}(I)$ and $\operatorname{SC}\left(I_{\text {res }}\right)$ are very different for the Bragg angle and the non-Bragg angles, the statistical properties in these two cases will also be very different. Thus, we will discuss these two cases separately.

\section{A. Statistics of the reflected field in the non-Bragg angles}

In the non-Bragg direction, we again find that the statistical properties of $E(\theta)$ and $\delta E(\theta)$ are very similar at different angles. Here, we take $\theta=174^{0}$ as an example. For the case of complete disorder, the distribution functions of the normalized amplitude $A=E(\theta) / \sigma$ and the phase of the reflected field are presented in Figs. 6(a) and 6(b), respectively. Here $2 \sigma^{2}=\left\langle|E(\theta)|^{2}\right\rangle-|\langle E(\theta)\rangle|^{2}$. The symbols "squares" and "circles" denote the results of the total and residual fields. The same symbols will be used in all other cases discussed below. Very little difference in the amplitude distributions of the residual and total fields indicates a negligible coherent component in this case. If we use the parameter $k=|\langle E(\theta)\rangle| / \sigma$ [or $k^{2}=2 a /(1-a)$ with $\left.a=I_{c} /\langle I\rangle\right]$ to represent the amount of the coherent component in the reflected field, ${ }^{18}$ the value of $k$ in this case is 0.242 according to the simulation result. Since the phase distribution of the residual field [circles in Fig. 6(b)] is constant, indicating a complete randomization of the phase, the RPS should give a good description of these data. The analytical distribution functions for the amplitude and phase obtained from RPS are, respectively, ${ }^{17,18}$

$$
P(A, k)=A \exp \left(-\frac{A^{2}+k^{2}}{2}\right) I_{0}(A k)
$$

and

$$
\begin{aligned}
P(\varphi, k)= & \frac{1}{2 \pi} \exp \left(-\frac{k^{2}}{2}\right)+\frac{k \cos \varphi}{\sqrt{2 \pi}} \\
& \times \exp \left(-\frac{(k \sin \varphi)^{2}}{2}\right) \operatorname{erf}(k \cos \varphi),
\end{aligned}
$$

where $I_{0}(x)$ is a modified Bessel function of the first kind of zero order, and $\operatorname{erf}(x)$ is an error function. By using the simulated value of $k=0.24$ in the above equations we obtain two solid lines in Figs. 6(a) and 6(b). Indeed, they overlap well with the simulated data of the total field. By taking $k$ $=0$ in the above equations, we obtain two dashed lines. They also agree well with the simulated data of the residual field. The dashed line in Fig. 6(a) represents Rayleigh statistics. The presence of a small coherent component makes the total intensity distribution very close to the Rayleigh distribution as indicated in Fig. 4(a). Although the intensity distribution of the total field is close to the Rayleigh distribution, its phase distribution is not completely random due to the presence of a small coherent component. Such a sensitive dependence of the phase distribution of the total field on the presence of a small coherent part has been also been observed in transmitted waves. ${ }^{18,19}$

For the case of small randomness with $d r=0.2$, the corresponding results are shown in Figs. 7(a) and 7(b). In this case, the value of $k$ is 1.70. Again, the RPS gives a very good description of the simulated data. Due to the presence of a larger coherent part (or higher value of $k$ ), the distribution of the total field [squares and the solid line in Fig. 7(a)] is very different from that of the residual field [circles and the dashed line in Fig. 7(a)]. The latter again has the Rayleigh distribution due to the complete phase randomization [circles and the dashed line in Fig. 7(b)]. Thus, we find a deviation from Rayleigh statistics in the total field when randomness is reduced as indicated in Fig. 4(b).

When the randomness is further reduced, in Figs. 8(a) and 8 (b), we show the results of the case $d r=0.1$ In this case the value of $k$ is increased to 4.17. Compared to Fig. 7, very similar behavior is observed in Fig. 8 except that there exists a small angular dependence in the phase distribution of the 

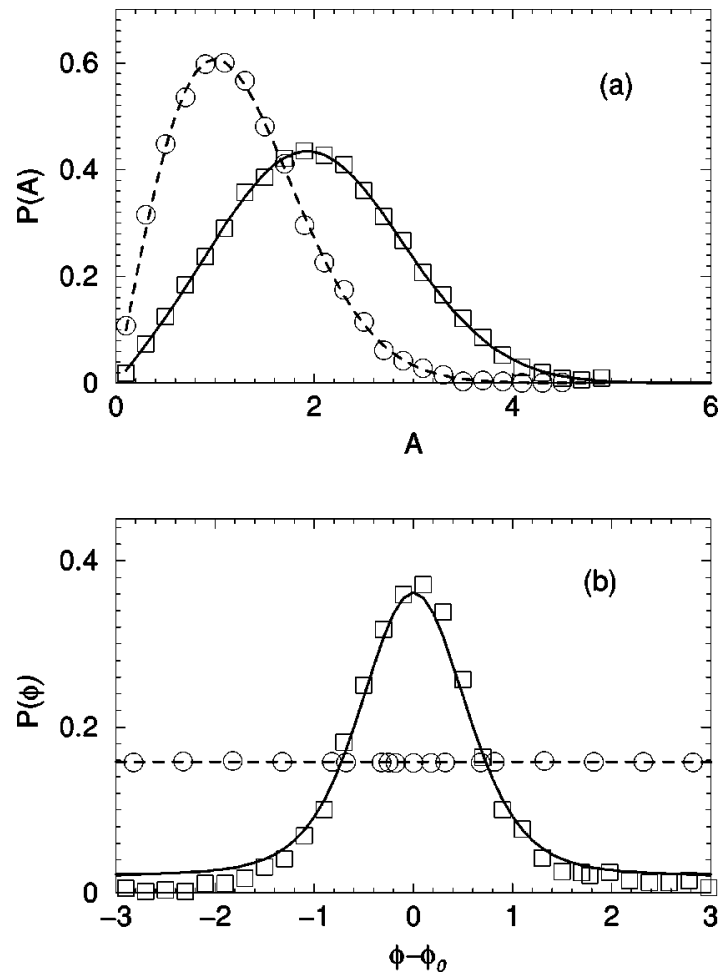

FIG. 7. Distributions of the normalized amplitude (a) and phase (b) of the total field (squares) and the residual field (circle) in the non-Bragg direction for small random system with $d r=0.2, R$ $=0.3$, and $f=0.44$. Solid lines and dotted lines are the theoretical predictions using the random-phasor-sum model.

residual field [circles in Fig. 8(b)]. Even the phase of the the residual field is not completely randomized, and the distribution of the total field still follows the prediction of the RPS. The amplitude distribution of the residual field still obeyes Rayleigh statistics. The validity of the RPS in the presence of a small nonuniformity in the phase distribution of the residual field has also been reported in the transmitted waves. $^{18,19}$

Thus, in the case of non-Bragg angles, the crossover behavior from the non-Rayleigh to Rayleigh distributions as a function of the degree of disorder is very similar to that of ballistic to diffusive wave propagation in transmitted waves. The reason for such a similarity is rather simple. In our system, the increase in randomness actually increases the density of the localized states in the system, which, in turn, suppresses the evanescent nature of the wave propagation and, therefore, increases the effective sample thickness for the wave propagtion. In fact, our Figs. 6-8 are very similar to Figs. 2 and 3 of Ref. 18. When the randomness is further reduced from $d r=0.1$, the value of $\mathrm{SC}(I)$ is reduced. However, the value of $\operatorname{SC}\left(I_{\text {res }}\right)$ remains close to 1 . This seems to indicate that the RPS is still valid and the amplitude distribution of the residual field is the Rayleigh type. Indeed, this is what we have found. In Fig. 9, we show various distribution functions for the case of $d r=0.03$. They are very similar to the case of $d r=0.1$ shown in Fig. 8, except that the phase distribution of the residual field is a little bit more nonuniform. The value of $k$ in this case is 14.03 .
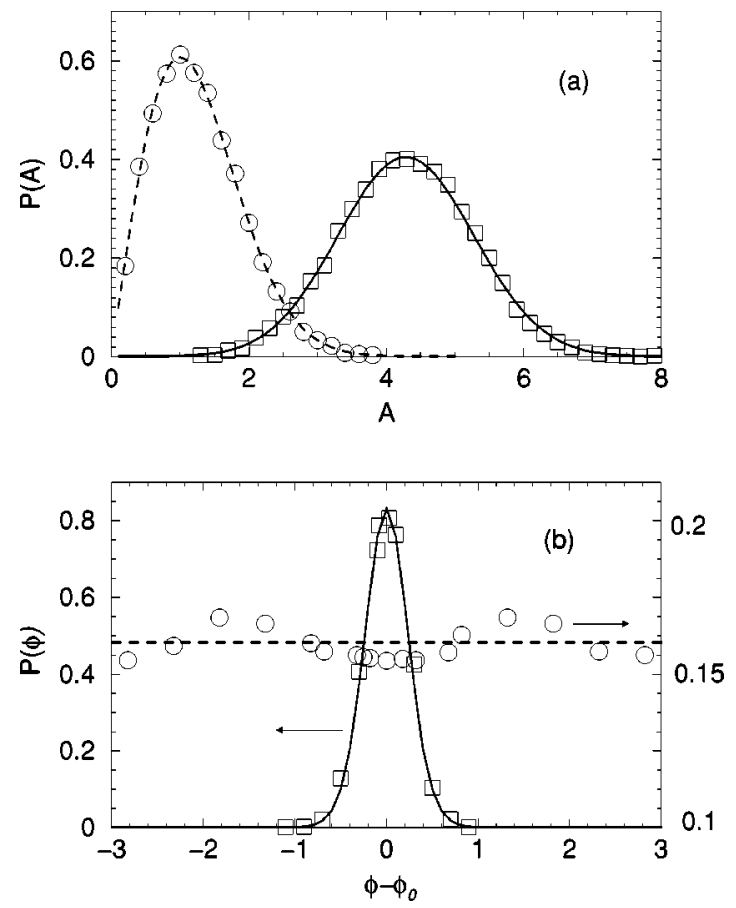

FIG. 8. Distributions of the normalized amplitude (a) and phase (b) of the total field (squares) and the residual field (circle) in the non-Bragg direction for a small random system with $d r=0.1, R$ $=0.3$, and $f=0.44$. Solid lines and dotted lines are the theoretical predictions using the random-phasor-sum model.
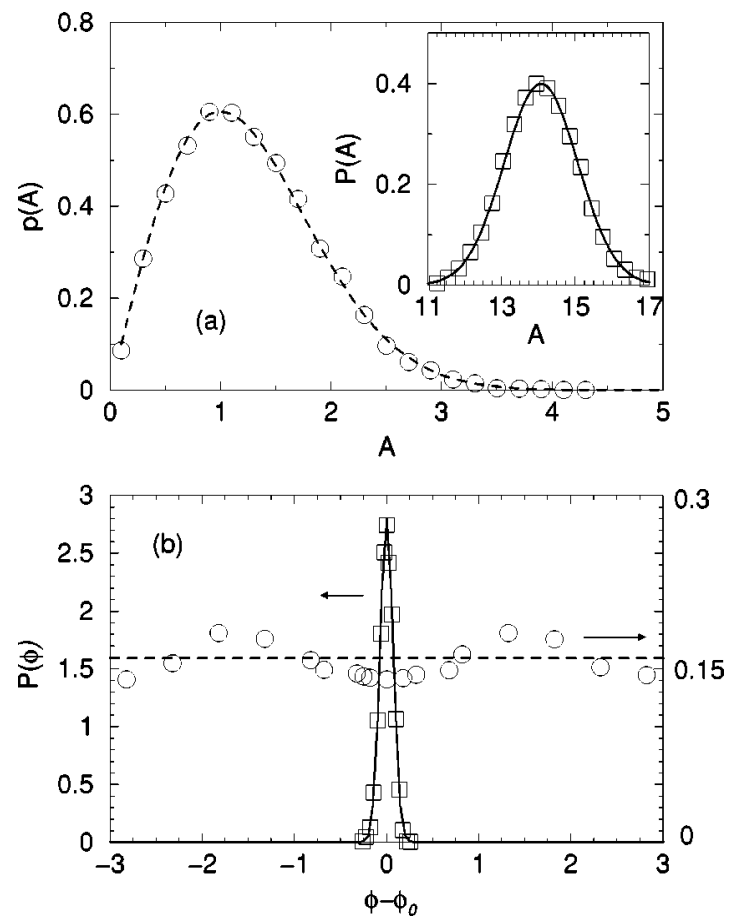

FIG. 9. Distributions of the normalized amplitude (a) and phase (b) of the total field (squares) and the residual field (circle) in the non-Bragg direction for small random system with $d r=0.03, R$ $=0.3$, and $f=0.44$. Solid lines and dotted lines are the theoretical predictions using the random-phasor-sum model. 

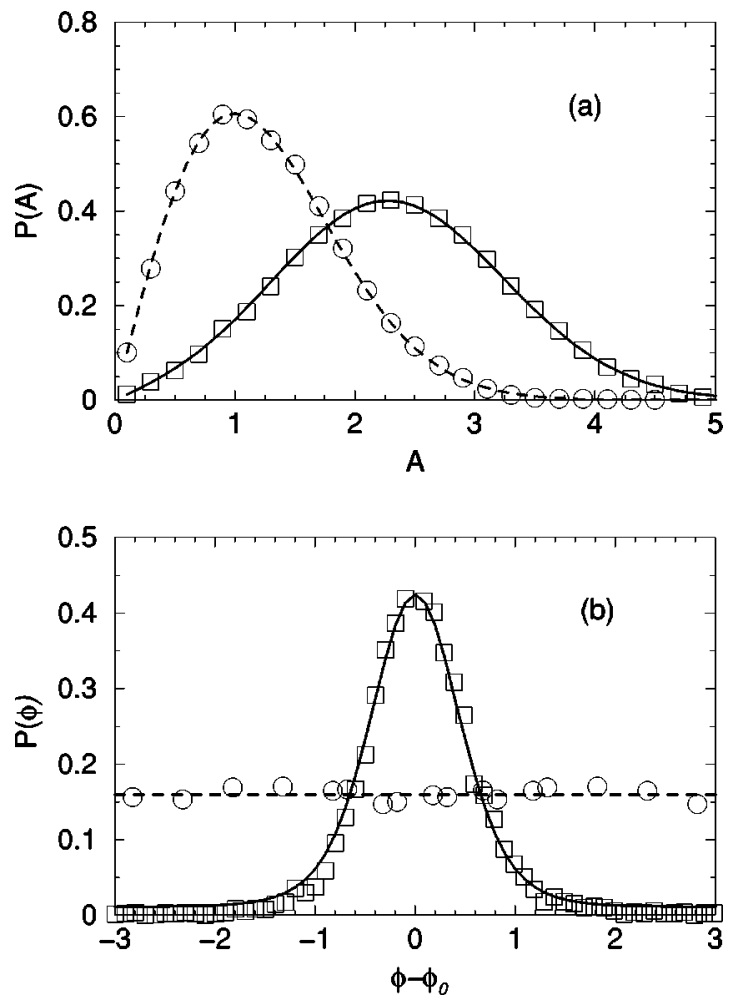

FIG. 10. Distributions of the normalized amplitude (a) and phase (b) of the total field (squares) and the residual field (circle) in the Bragg direction for small random system with $d r=0.2, R$ $=0.3$, and $f=0.44$. The results of the total field are presented in the inset of (b). Solid lines and dotted lines are the theoretical predictions using the random-phasor-sum model.

Here, we would like to emphasize again that the validity of the RPS shown in Figs. 6-9 lies in the certain degree of phase randomization in the residual field. This can also be seen from the closeness of the value of $\operatorname{SC}\left(I_{\text {res }}\right)$ to one as indicated in Fig. 5 (squares). This is also the reason that all the amplitude distributions of the residual field (circles) found in Figs. 6(a)-9(a) are close to the Rayleigh distribution (dashed line). However, this is not true for the Bragg angle. In Fig. 5, the values of $\operatorname{SC}\left(I_{\text {res }}\right)$ are much greater than one except in the case of complete randomness. Thus, it is expected that the RPS will fail to describe the case of the Bragg angle when randomness is small.

\section{B. Statistics of the reflected field in the Bragg angle}

In contrast to the case of the non-Bragg angle, the statistical distributions in the Bragg angle are more complicated. For the case of complete randomness, the results are shown in Fig. 10. It is clear that the RPS is still valid in this case. This is not surprising from the small values of $k=2.07$ and $\operatorname{SC}\left(I_{\text {res }}\right) \approx 1$ (filled circles in Fig. 5). It is also clear that, even for the case of complete randomness, the amplitude distribution of the total field is not a Rayleigh distribution. This is due to the presence of a large coherent component in the total field, which also makes the value of $\operatorname{SC}(I)$ smaller than one as shown by the filled diamonds in Fig. 5 .
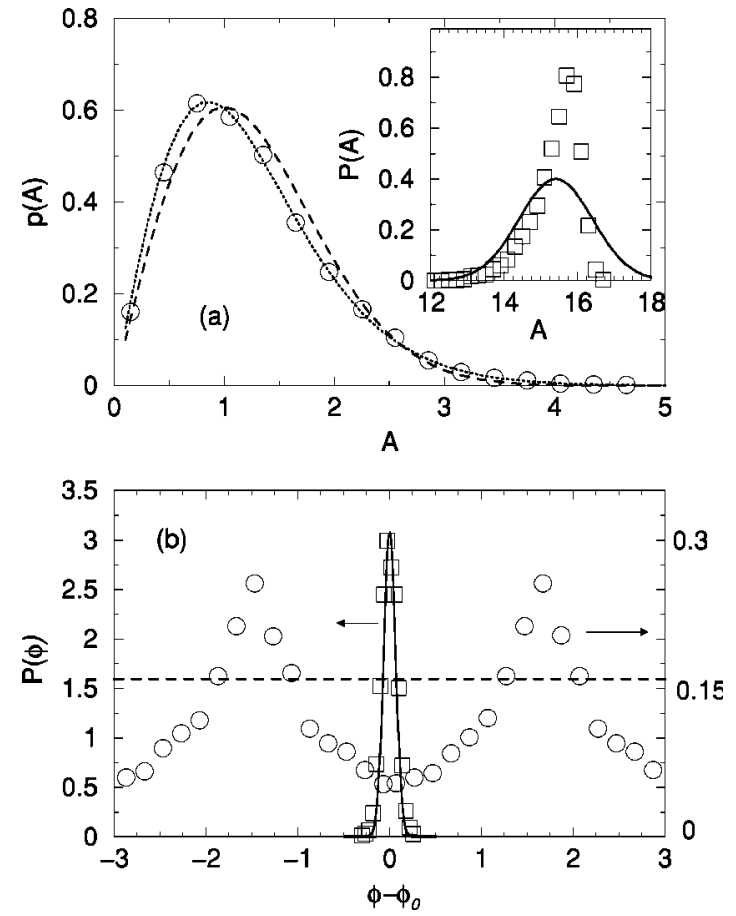

FIG. 11. Distributions of the normalized amplitude (a) and phase (b) of the total field (squares) and the residual field (circle) in the Bragg direction for small random system with $d r=0.2, R$ $=0.3$, and $f=0.44$. The results of the total field are presented in the inset of (b). Solid lines and dotted lines are the theoretical predictions using the random-phasor-sum model.

When the disorder is reduced, we see that the value of $\mathrm{SC}\left(I_{\text {res }}\right)$ increases monotonously, whereas the value of $\mathrm{SC}(I)$ decreases rapidly (Fig. 5). Since $\operatorname{SC}\left(I_{\text {res }}\right)>1$, it is expected that the amplitude distribution of the residual field will deviate from the Rayleigh distribution. This is indeed what we have found. In Fig. 11, we show that results for the case of $d r=0.2$. A clear deviation in the circles from the dashed line in Fig. 11(a) can be seen. This deviation is also reflected from the much larger nonuniformity in the phase distribution of the residual field as indicated by the circles in Fig. 11(b). Similar behavior has been seen in the transmitted waves and is attributed to the incomplete randomization of the phase in the residual field so that the speckle pattern is not completely devoloped. ${ }^{19}$ In this case, the RPS cannot describe the statistical distribution of the total field. Indeed, in the inset of Fig. 11(a), we see large deviations between the simulated data (squares) and the predication of Eq. (4) (the solid line), although certain agreement between the two in the phase distribution remains, i.e., the agreement between the squares and the solid line in Fig. 11(b). The value of $k$ in this case is 15.36, indicating a large coherent component in the total field. It is interesting to point out that the value of $k$ $(=15.36)$ in this case is not very different from that in the case of non-Bragg angles with $d r=0.1$, i.e., $k=14.03$. Both cases show a large coherent part in the scattered intensities. However, their values of $\mathrm{SC}\left(I_{\text {res }}\right)$ are very different. As will be discussed later, the large value of $\operatorname{SC}\left(I_{\text {res }}\right)$ in the case of the Bragg angle is due to the single mode excitation, whereas, this is not the case for the non-Bragg angles. 

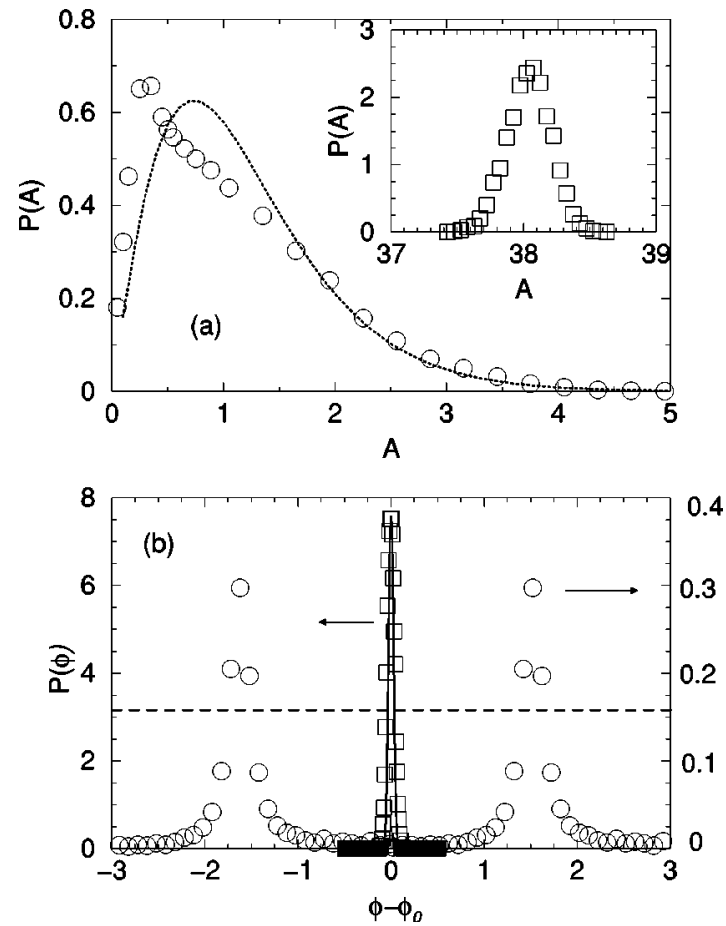

FIG. 12. Distributions of the normalized amplitude (a) and phase (b) of the total field (squares) and the residual field (circle) in the Bragg direction for small random system with $d r=0.1, R$ $=0.3$, and $f=0.44$. The results of the total field are presented in the inset of (b). Solid lines and dotted lines are the theoretical predictions using the random-phasor-sum model.

It has been suggested that when $\mathrm{SC}>1$, one can use $K$ distribution to describe the amplitude distribution of the residual field. When SC approaches one, the Rayleigh distribution is recovered. The $K$ distribution was derived to describe the satistics of the partially developed speckle intensities and has the form ${ }^{17,19}$

$$
P(A)=\frac{2 \sqrt{2 M}}{\Gamma(M)}\left(\frac{M A^{2}}{2}\right)^{M / 2} K_{M-1}(A \sqrt{2 M}),
$$

where $K_{\nu}(x)$ is the modified Bessel function of the second kind of order $\nu$ and $2 / M=\operatorname{SC}\left(I_{\text {res }}\right)^{2}-1$. Using the value of $\mathrm{SC}\left(I_{\text {res }}\right)=1.18$ in Eq. (6), we obtain the $K$ distribution and plot it as the dotted line in Fig. 11(a). The excellent agreement between the dotted line and the circles indicates the validity of the $K$ distribution.

In Fig. 12, we show the results for the case of $d r=0.1$. In this case, $\operatorname{SC}\left(I_{\text {res }}\right)=1.34$. A clear deviation from the $K$ distribution is seen in Fig. 12(a), in which the dotted line does not overlap with the circles. In this case, the phase distribution of the residual field, i.e, the circles in Fig. 12(b), is far from randomized and has two sharp peaks on each side of the phase of the coherent field. It should be pointed out that the total field is predominated by the coherent wave and only $0.1 \%$ of the total intensity comes from the scattered field. Finally, we would like to point out we have repeated the calculations for the case of oblique incidence. The results are similar to the case of normal incidence shown in Figs. 6-12.
The very different behaviors found in Figs. 5-12 between the Bragg and the non-Bragg angles can be understood in the following way. In the case of the Bragg angle, the coherent part of the reflected field comes from the "mirror" reflection of the incident wave. From Fig. 3, it is clear that the dominant part of the incident wave is a plane wave. This is similar to the case of the single-mode incoming wave discussed in Ref. 19. For single mode incident, it has been pointed out that the fluctuations of the field in the incident mode are much larger than those of the other modes. As a result, a departure from Rayleigh statistics in the intensity of the residual field would be expected. ${ }^{18}$ Thus, large values of $\operatorname{SC}\left(I_{\text {res }}\right)$ have been found in the case of the Bragg angle. However, this is not true for the non-Bragg angles. In this case the coherent part of the field is a result of wave diffraction due to the finite beam width of the incident wave. It appears with small intensity and angular fluctuations in Fig. 3. Thus, for the non-Bragg angles, the phase randomization of the residual field is very easily achieved even with the presence of small randomness. This is why the value of $\mathrm{SC}\left(I_{\text {res }}\right)$ is always close to one, independent of the randomness. Here, we would like to mention that, for the case of complete randomness, the structure of the coherent backscattering cone has been studied recently. ${ }^{22,23}$ Since, in this case, $\operatorname{SC}(I)$ is also close to one, the coherent part of the intensity becomes negligible in the scattered waves. A complete phase randomization without a coherent part indicates that the wave propagation near the input surface can be described by a diffusive propagation with the localization length as the upper cutoff length. In fact, it has been shown that such a simple description is capable of explaining the structure of the coherent backscattering cone obtained by numerical simulations. ${ }^{23}$

\section{CONCLUSIONS}

By using the multiple-scattering method, we have studied in detail the statistics of the reflected speckle intensities arising from the localized states inside the gap of disordered photonic crystals in two dimensions. We have also studied the statistics of the Lyapunov exponent of the transmitted waves. Similar to the case of disordered photonic crystals in one dimension, two types of localized states are found depending on the degree of disorder, and the frequency inside the gap. Our simulation results indicate that the reflection statistics depend on whether or not the localized states are the normal type. They also depend on whether or not the reflected angles are in the Bragg angle. For the non-Bragg angles, we found that the intensity distribution arising from the normal-type localized states follows Rayleigh statistics, in agreement with random matrix theory. However, the deviations from Rayleigh statistics were found for the case of the second type of localized states.

The crossover behavior from non-Rayleigh to Rayleigh statistics were studied as a function of the degree of disorder at some fixed frequency inside the gap. By separating the field into the coherent and diffuse parts, we have studied the statistics of field and phase distributions for both diffuse and total fields as well as their speckle contrasts. It is found that 
the crossover behavior is very similar to behavior of ballistic-to-diffusive wave propagation for transmitted waves and can be described by the RPS.

For the case of the Bragg scattering angle, the nonRayleigh statistics are found for both kinds of localized states. Furthermore, the statisics are sensitive to the degree of disorder. The RPS is valid only at large randomness. As the randomness is reduced, the $K$ distribution becomes a valid description of the residual field. When the randomness is further reduced, even the $K$ distribution becomes invalid. The different behaviors between the Bragg and non-Bragg angles are because the Bragg angle reflection is a result of singlemode excitation, whereas, this is not true for true for nonBragg angles.

\section{ACKNOWLEDGMENTS}

This work was supported by Hong Kong Research Grants Council Grant No. HKUST 6163/01P.
*Author to whom correspondence should be addressed. Electronic address: phzzhang@ust.hk

${ }^{1}$ See, for example, Scattering and Localization of Classical Waves in Random Media, edited by P. Sheng (World Scientific, Singapore, 1990); P. Sheng, Introduction to Wave Scattering, Localization and Mesoscopic Phenomena (Academic Press, New York, 1995); M. C. W. van Rossum and T. M. Nieuwenhuizen, Rev. Mod. Phys. 71, 313 (1999).

${ }^{2}$ E. Wolf, Phys. Rev. Lett. 56, 1370 (1986); B. Shapiro, ibid. 57, 2168 (1986); M. Stephen and G. Cwilich, ibid. 59, 285 (1987); S. Feng, C. Kane, P. A. Lee, and A. D. Stone, ibid. 61, 834 (1988); I. Freund, M. Rosenbluh, and S. Feng, ibid. 61, 2328 (1988); N. Garcia and A. Z. Genack, ibid. 63, 1678 (1989); R. Berkovits, M. Kaveh, and S. Feng, Phys. Rev. B 40, 737 (1989); M. P. van Albada, J. F. de Boer, and A. Lagendijk, Phys. Rev. Lett. 64, 2787 (1990).

${ }^{3}$ A. Z. Genack and N. Garcia, Europhys. Lett. 21, 753 (1993); J. F. de Boer, M. C. W. van Rossum, M. P. van Albada, Th. M. Nieuwenhuizen, and A. Lagendijk, Phys. Rev. Lett. 73, 2567 (1994); M. Stoytchev and A. Z. Genack, ibid. 79, 309 (1997).

${ }^{4}$ Th. M. Nieuwenhuizen and M. C. W. van Rossum, Phys. Rev. Lett. 74, 2674 (1995); E. Kogan and M. Kaveh, Phys. Rev. B 52, R3813 (1995).

${ }^{5}$ I. Edrei et al., Phys. Rev. Lett. 62, 2120 (1989); N. Shnerb and M. Kaveh, Phys. Rev. B 43, 1279 (1991); E. Kogan et al., ibid. 48, 9404 (1993); E. Kogan and M. Kaveh, ibid. 51, 16400 (1995).

${ }^{6}$ P. A. Mello, E. Akkermans, and B. Shapiro, Phys. Rev. Lett. 61, 459 (1988).

${ }^{7}$ C. W. J. Beenakker, Rev. Mod. Phys. 69, 731 (1997).

${ }^{8}$ S. A. van Langen, P. W. Brouwer, and C. W. J. Beenakker, Phys. Rev. E 53, R1344 (1996).

${ }^{9}$ A. Garcia-Martin, J. A. Torres, J. J. Saenz, and M. Nieto-
Vesperinas, Phys. Rev. Lett. 80, 4165 (1998).

${ }^{10}$ A. Garcia-Martin, T. Lopez-Ciudad, and J. J. Saenz, Phys. Rev. Lett. 81, 329 (1998).

${ }^{11}$ L. I. Deych, D. Zaslavsky, and A. A. Lisyansky, Phys. Rev. Lett. 81, 5390 (1998); L. I. Deych, A. A. Lisyansky, and B. L. Altshuler, ibid. 84, 2678 (2000); Phys. Rev. B 64, 224202 (2001).

${ }^{12}$ Yu. A. Vlasov, M. A. Kaliteevski, and V. V. Nikolaev, Phys. Rev. B 60, 1555 (1999).

${ }^{13}$ See, for example, Photonic Band Gaps and Localization, edited by C. M. Soukoulis (Plenum, New York, 1993); J. Opt. Soc. Am. B 10, 208 (1993); Photonic Band Gap Materials, edited by C. M. Soukoulis (Kluwer, Dordrecht, 1996); J. D. Joannopolous, R. D. Meade and J. N. Winn, Photonic Crystals (Princeton University, Princeton, 1995).

${ }^{14}$ S. John, Phys. Rev. Lett. 58, 2486 (1987).

${ }^{15}$ R. Dalichaouch et al., Nature (London) 354, 53 (1991).

${ }^{16}$ Z. Q. Zhang et al., Phys. Rev. Lett. 81, 5540 (1998).

${ }^{17}$ J. W. Goodman, in Laser Speckle and Related Phenomena, edited by J. C. Dainty (Springer-Verlag, Berlin, 1984); J. W. Goodman, Statistical Optics (Wiley, New York, 1985); J. Ohtsubo and T. Asakura, Opt. Commun. 14, 30 (1975); H. Fujii and T. Asakura, ibid. 11, 35 (1974); H. M. Pederson, ibid. 12, 156 (1974).

${ }^{18}$ A. A. Chabanov and A. Z. Genack, Phys. Rev. E 56, R1338 (1997).

${ }^{19}$ A. Garcia, J. J. Saenz, and M. Nieto-Vesperinas, Phys. Rev. Lett. 84, 3578 (2000).

${ }^{20}$ L. M. Li and Z. Q. Zhang, Phys. Rev. B 58, 9587 (1998).

${ }^{21}$ E. Kogan and M. Kaveh, Phys. Rev. B 51, R16 400 (1995).

${ }^{22}$ J. Huang, N. Eradat, M. E. Raikh, Z. V. Vardeny, A. A. Zakhidov, and R. H. Baughman, Phys. Rev. Lett. 86, 4815 (2001); A. Yu. Sivachenko, M. E. Raikh, and Z. V. Vardeny, Phys. Rev. B 63, 245103 (2001).

${ }^{23}$ X. Zhang and Z. Q. Zhang, Phys. Rev. B 65, 155208 (2002). 